\title{
Percentage and Intensity in Parasite That Attack Common Carp Fish Cyprinus carpio from Alforat River Breeding Fish Cages Al-Mussayab/Iraq
}

\author{
Hussein Takheal Hussein \\ Dept. of Animal production, Technical Inst. Mussayab, Al-Furat Al-Awsat technical \\ university \\ Husseintakheal@gmail.com
}

Keywords: Cyprinus carpio, Cages, AL-Mussayab.

\begin{abstract}
84 samples of common carp Cyprinus carpio that bird in cages of fishes birding in AlForat River Al-Mussayab was examined for a period of January until June 2017 .

The fishes was examined for the presence of Ichthyophthirius multifiliis parasite on the skin, Fins and gills.

The percentage and total mean intensity infection\%14.28, 5.6 ‘\% 20.23, $6.2, \% 27.3811 .5$ respectively the monthly changes was Studied and the part of the fish body was chosen according to sex and we recording the highest percentage of infection from April 53.33\% and highest intensity in August were the percentage was $21.6 \%$.

There was no recording of differences in chosen of site of infection and also in sex and host either in skin, Fins and gills.
\end{abstract}

Ichthyophthirius multifiliis Fouquet, 1876 نسبة وشدة الإصابة بالطفيلي لأسماك الكارب الاعتيادي Cyprinus carpio من أقفاص تربية الأسماك في نهر الفرات.

$$
\text { المسيب/العز اق من }
$$

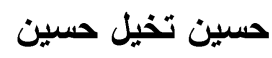

قسم الإنتاج الحيواني، المعهد التقني/المسيب، جامعة الفرات الاوسط التقنية

تم فحص عه نموذج من اسماك الكارب الاعتيادي Cyprinus carpio المرباة فـــي أقفــاص تربيــة

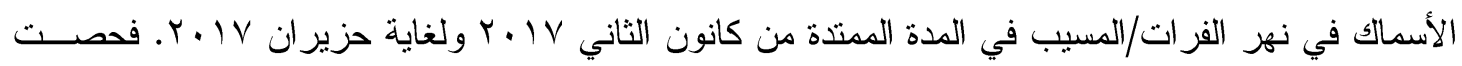

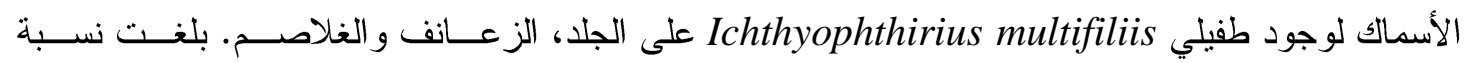

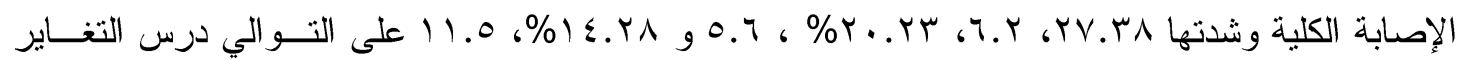


الثهري واختيار موقع الإصـابة في جسم الأسماك وبحسب الجنس تم تسحيل أعلى نسبة إصــابة فــي شـــر

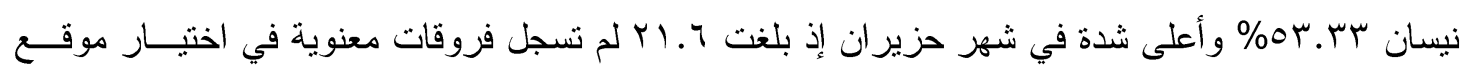

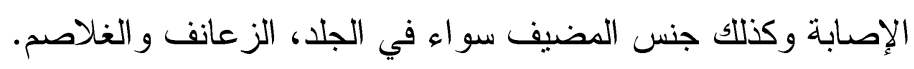

الكلمات المفتاحية: الكارب الاعتيادي، أقفاص، المسيب

المقدمة

يعد الاستزراع السمكي من الرو افد المهمة في العديد من دول العالم وله اثر كبير في الأبعــاد الغذائيــة و الاقتصادية و الاجتماعية [1] وتتعدد طبيعة أنشطة الاستزراع السمكي في الوطن العربي تبعا للمو ارد المتاحة

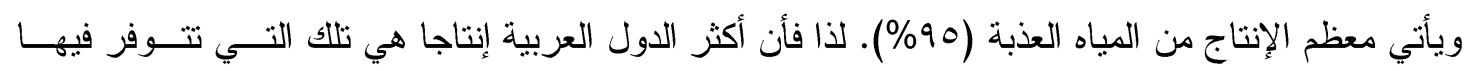

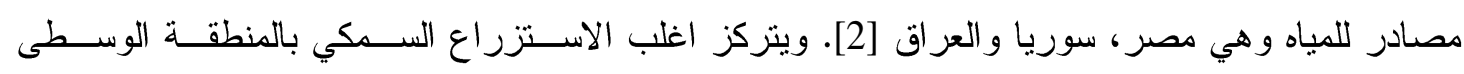

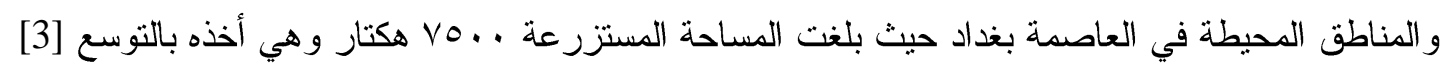

إن إصـابة الأسماك بالطفيليات تسبب خسائر اقتصادية مباثـــرة [4] و ان الإصـــابات بالطفيليــات منهـــا

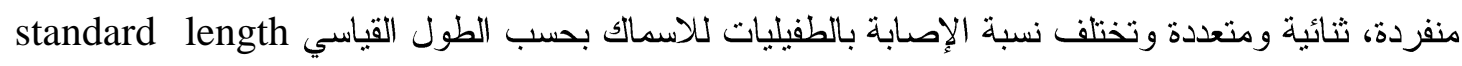

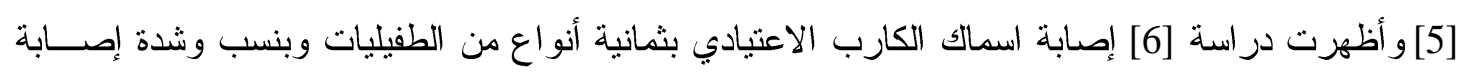

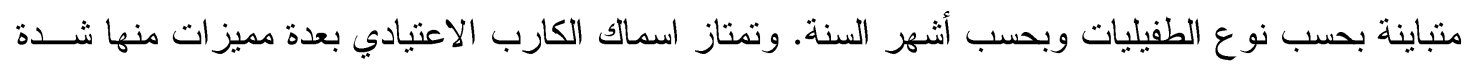

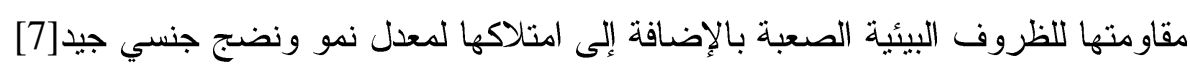
طفيلي Ciliates دو شن الهدب د. multifilis دائري يصيب معظم أنواع الأسماك مثل الكارب

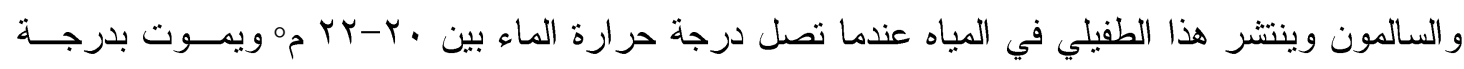

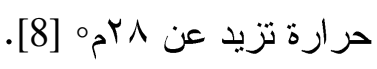

تسبب طفيليات هذا الجنس ضرر اللأسماك في كل أرجاء العالم أكثر من أي طفيلــي أخــر [9] ويعـده

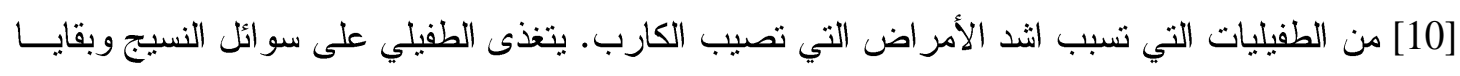

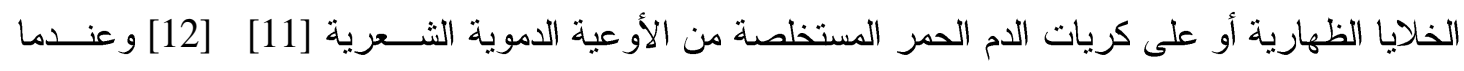

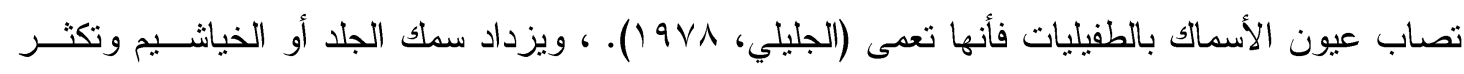
المو اد المخاطية حوله ويظهر في شكل نقط بيضاء، وعند الإصابة الثديدة نزداد النقط البيضاء وتبدو الأسماك

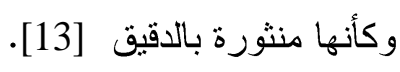

صمم في الدر اسة الحالية حساب نسبة وشدة الإصابة بطفيلي I. multifilis في اسماك الكارب الاعتيادي

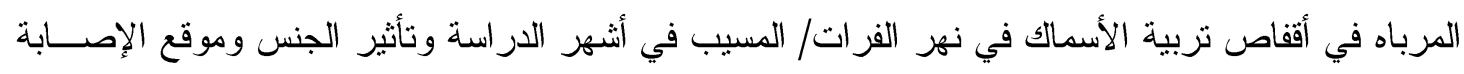
في نسبة الإصابة وشدتها. 


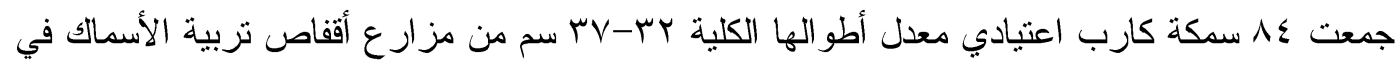

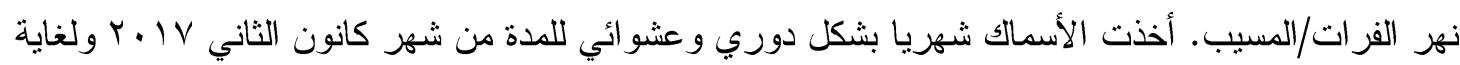

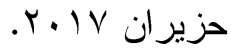

نقلت الأسماك حية إلى المختبر وعين جنسها ثم قتلت مباتشرة بعد ذلك بطريقة الضرب بقطعــة خشــبية على الر أس. فحصت الأسماك خارجيا بالعين المجردة او استخدمت عدسة مكبرة بحثا عن طفيلي

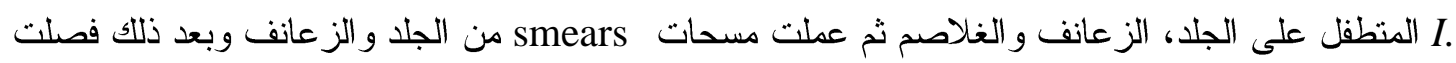
الغلاصم من تجويفها الغلصمي ووضعت في طبق بتري حاوي على ماء حنفية وفحصت بالعين المجردة أو لا

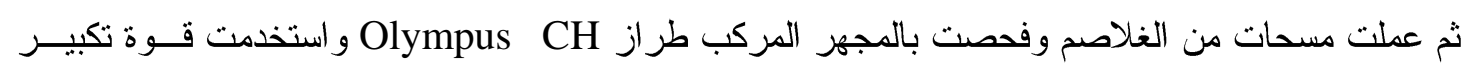

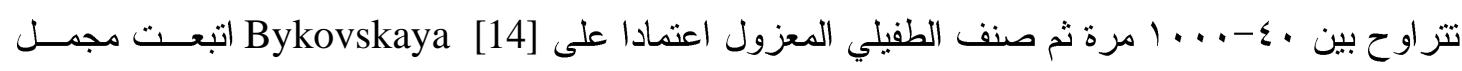

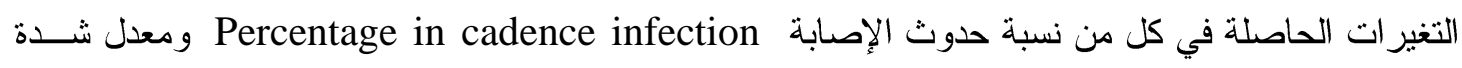
الإصابة Mean intensity infection العنمادا على[15] (1974) .

التحليل الإحصائي لغرض مقارنة التغيرات الحاصلة في نسبة حدوث الاصابة ومعدل شدة الاصابة في أثنهر الدراسة وتأثير جنس المضيف واختيار موقع الاصابة بالطفيلي Ichthyophthirius multifiliis فقد تم إجر اء اختبار مربع كاي Chi-square على وفق جداول الاحتمالات Contingency tables الموضحة في [16].

\section{النتائج و المناقشة}

بينت نتائج الدراسة الحالية إصابة اسماك الكارب الاعتيادي بالطفيلي I. multifiliis. وشهدت الإصــابة

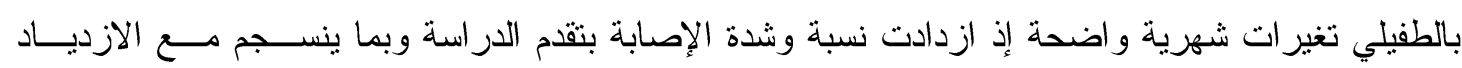

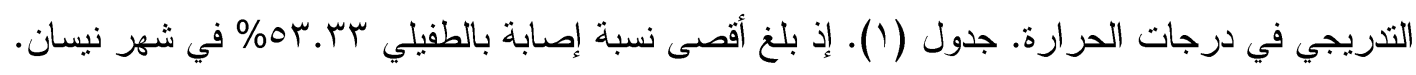

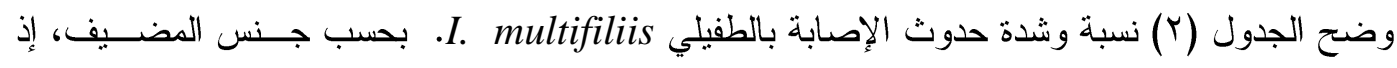

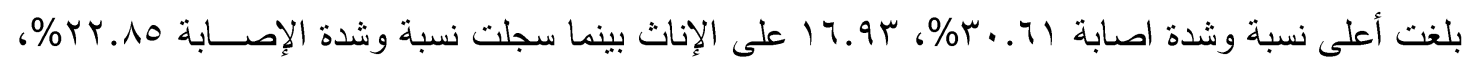
IV.rV

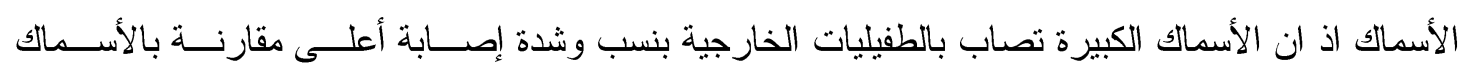

الصغيرة [6]

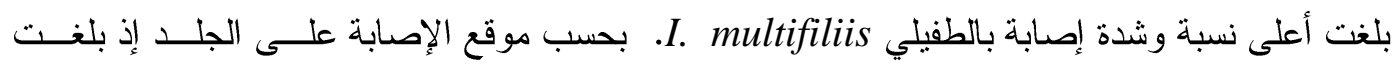
^r.r.

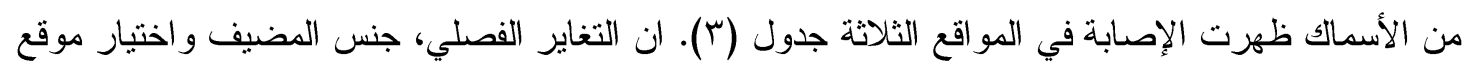

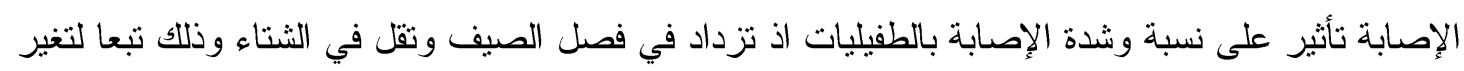

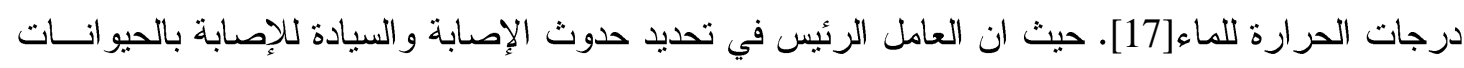
الابتدائية والتي تتكاثز في الماء الذي درجة حرارته عاليه[18] و هذا يتفق مع ماتوصل اليه [19] في دراسته 


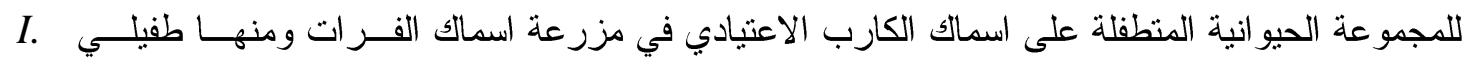
هultifiliis في شهر شباط. ويسبب هذا الطفيلي مرض النقطة البيضاء White Spots Disease إذ يخترق الطفيلي جلد الأسماك و الخياشيم ويقوم الجسم بتكوين العديد من الخلايا حول الطفيلي

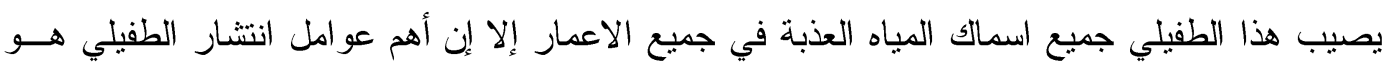

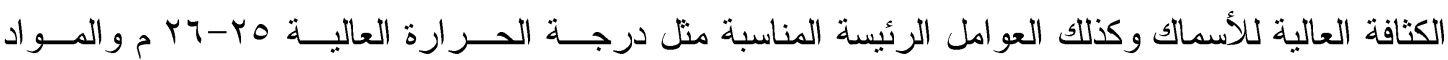

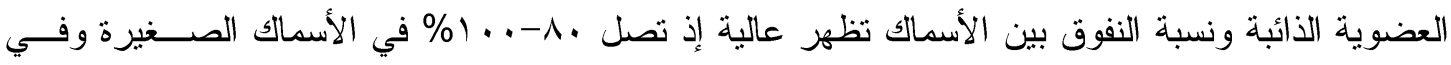

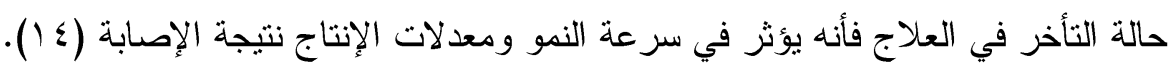

أظهرت نتائج التحليل الإحصائي عدم وجود فروق معنوية عند مستوى احتمالية ه... جدول (ع) فــي

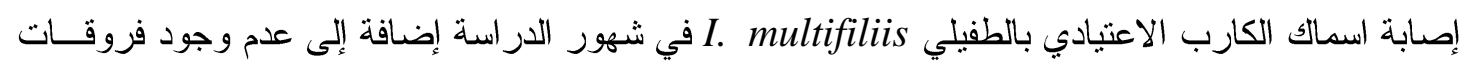
معنوية في الجنس جدول (0). ويرجع سبب ذلك إلى تو اجد الأسماك في مساحة صغيره وكثافة عاليــهـ فــي

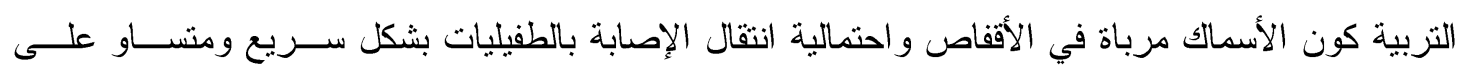
الأسماك سواء أكانت ذكور ا أم إناث وقلة مسافة الحركة للأسماك.

\begin{tabular}{|c|c|c|c|c|c|}
\hline شدة الإصـابة & عدد الطفيليات & $\begin{array}{c}\text { نسبة الإصابة } \\
\text { \% } \\
\text { \% }\end{array}$ & عدد الأسماك & عدد الأسماك (1): سببه & الشهر \\
\hline 11.0 & $\leq 7$ & YT.74 & $\varepsilon$ & 10 & كانون الثاني V Y. V \\
\hline 1.0 & iv & $10 . r \Lambda$ & r & r & شباط \\
\hline $1 \varepsilon$ & 07 & YE.OV & $\varepsilon$ & $1 \leq$ & اذار \\
\hline r..YO & IV. & 53.33 & $\wedge$ & 10 & نيسان \\
\hline 19.0 & $r q$ & 11.11 & r & 11 & مايس \\
\hline r.. & 70 & 11.20 & r & 17 & حزير ان \\
\hline 17.0 & rar & rV.rA & rr & $\wedge \varepsilon$ & المجموع \\
\hline
\end{tabular}

I. multifiliis جدول (r): تأثير جنس المضيف في نسبة وشدة الإصابة بالطفيلي

\begin{tabular}{|c|c|c|c|c|c|}
\hline شدة الإصـابة & عدد الطفيليات & نسبة الإصـابة \% & عدد الأسماك & عدد الأسماك & المضبف \\
\hline IV.rV & 139 & YY.AO & $\wedge$ & o & ذكور \\
\hline 17.94 & ros & $r \cdot .7)$ & 10 & $\leq 9$ & إث \\
\hline IV & س ب & rV.rN & rr & $\Lambda \varepsilon$ & المجموع \\
\hline
\end{tabular}


I. multifiliis جدول (َ): اختيار الموقع في الغلاصم و الزعانف والجلا بالطفيلي

\begin{tabular}{|c|c|c|c|c|c|}
\hline شدة الإصـابة & عدد الطفيليات & نسبة الإصابة & عدد الأسماك & عدد الأسماك & موقع الإصـابة \\
\hline T.Y & $1 \leq \mu$ & rV.r & rr & $\Lambda \varepsilon$ & الجلد \\
\hline 1.0 & $11 r$ & r. & IV & $\wedge \varepsilon$ & الزعانف \\
\hline \multirow[t]{2}{*}{11.0} & אזו & $1 \leq . Y \wedge$ & Ir & $\wedge \varepsilon$ & الغلاصم \\
\hline & & & تداخل في الإصابة * & $\Lambda \varepsilon$ & المجموع \\
\hline
\end{tabular}

* الاصابه في الغلاصم و الزعانف و الجلد

جدول (ء): نتائج التحليل الإحصائي لإصابة اسماك الكارب الاعتيادي بالطقيلي I. multifiliis حسب

\begin{tabular}{|c|c|c|c|c|}
\hline & & & & \\
\hline الجدولية X' & المحسوبة X2 & عدد الأسماك & عدد الأسماك غير & أثثهر الدر اسة \\
\hline \multirow{6}{*}{14.091} & \multirow{6}{*}{ V. II * } & $\varepsilon$ & 11 & كانون الثاني Y IV \\
\hline & & $r$ & 11 & شباط \\
\hline & & $\varepsilon$ & 1. & آذار \\
\hline & & $\wedge$ & V & نيسان \\
\hline & & r & 9 & مايس \\
\hline & & r & $1 \pi$ & حزيران \\
\hline
\end{tabular}

* فروق معنوية عند مستوى احتمالية ه. ...

جدول (•): نتائج التحليل الإحصائي لإصابة اسماك الكارب الاعتيادي بطفيلي I. multifiliis حسب

\begin{tabular}{|c|c|c|c|c|}
\hline \multicolumn{5}{|c|}{ الجنس. } \\
\hline الجدولية X & X المحسوبة X2 & عدد الأسماك & عدد الأسماك غير & الجنس \\
\hline \multirow[t]{2}{*}{0.991} & \multirow[t]{2}{*}{$\cdot V \leqslant V^{*}$} & $\wedge$ & rV & ذكور \\
\hline & & 10 & ए乏 & إناث \\
\hline
\end{tabular}

* فروق معنوية عند مستوى احنمالية ه.... 


\section{(المصادر}

[1] FAO (2004) Primary aquatic animal health care in rural. Small. Scal, aquaculture development. FAO, Tech. 406:55-61.

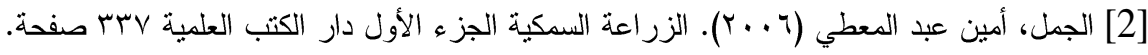

$$
\begin{aligned}
& \text { [3] المختار، مصطفى احمد (ب. . ب) طرق استزراع الأسماك في الاهوار العر اقية، المؤتمر العلمي الأول، مركز علوم البحار } \\
& \text { | (1) ا نيسان (ملخصات ابحاث). }
\end{aligned}
$$

[4] Mhaisen, F. T.(1989). Records of some fish Parasites from shatt AL-Arab revier and the north west of the Arab Gulf. Bull - Basrah Nat. Hist. Mus., 6(1):111-124 PP.

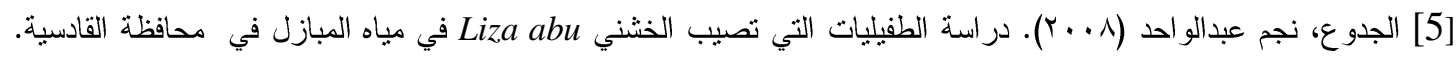

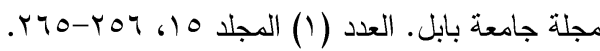

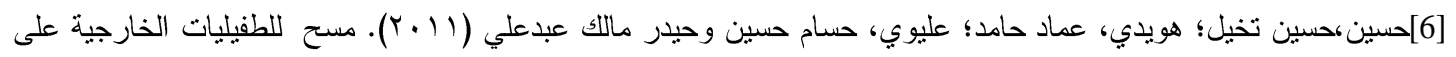

$$
\begin{aligned}
& \text { اسماك الكارب الاعتيادي Carpio Cyprinus في ثلاث مزارع سمكية في الإنكندرية/محافظة بابل. مجلة جامعة كربلاء }
\end{aligned}
$$

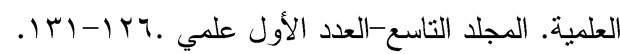

[7] Jeney, Z. and geney, G. (1995). Recent achievements in studies on disease of cdmmon carp. (Cyprinus carpio L.) Aguaculture. 129:397-420.

[8] اللوس، سناء بثير؛ الثيخ، محمد عادل عبدالرزاق وقيس يامور منصور (.99.). أساسيات علم الأسماك. دار الحكمة

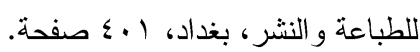

[9] Rogers, W.A.; Gaines, J. L. (1975). Lesions of protozoan disease in fish. Ribelin, W-E.; Migaki, G(Eds). PP. 117-141.

[10] Bauer, O.N.; Musselius, V.A.; \& strelkov, Yu. A. (1969). Diseases of pond fishes, Izdat. Kolos, Moscow, 220pp. (In Russian).

[11] Afanacev, V.E.; \& Yakovchuk. T. A(1971).Diseases of Industrial fishes. Kragnodar. Knees. Ezdat., 96 pp. (In Russian).

[12] Duijn, van. Jur. C. (1973) Diseases of fishes. lliffe Books. London, 3rd ed., 372pp.

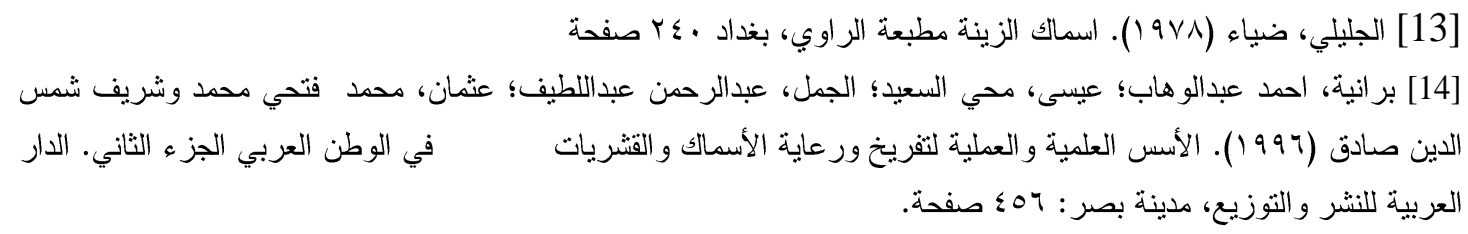

[15] Bykovskaya-pavlovskaya, I. E.; Gusev. A.V.; Dubinina, M. N.; Izyumova, N. A.; Smirnova, T. S.; Sokovskaya, I.L.; Shtein, G. A.; Shulman,. S.S. \& Epshtein, v.M. (1962). Key to parasites of freshwater fish of the U.S.S.R Akad. Nauk, S.S.S.R., Moscow: 727pp. (In Russian).

[16] Snedecor, G. W. and Cochran, W. G. (1974). Statical methods. $6^{\text {th }}$ edn., Iows state Univ. Press: $221 \mathrm{pp}$.

[17] Campbell, G.A. (1967). Statistics for biologists. Cambridge Univ. Press: 242pp. of fishes (Engl. Transl.) Oliver and Boyd Ltd., Edinburgh and London: 384 PP. 
[18] Bilal, Samir jawdat (2016). Seasonal distribution and site selection of Paradiplozoon barbi (Reichenbach-Klinke, 1951) infesting Cyprinidae macrostomum (Osteichthyes: Cyprinidae from Greater Zab river in Erbil- Kurdistan/Iraq. POLYTECHNIC Vol. 6No3, 463-473pp.

[19] Zargara, U,R.; Chishtia, M. Z.; Yousufa, A. R. and Fayaz, A. (2012). Infection level of monogenean gill parasite, Diplozoon Kashmirensis (Monogenea, Polyopisthocotylea) in the crucian carp Carassius carassius from lake ecosystems of an altered water quality: What factors do have an impact on the Diplozoon Kashmirensis Vet. Parasitol., 189:218-226.

$$
\begin{aligned}
& \text { [20]الزبيدي، علي بناوي (991)). دراسات حول المجموعة الحيوانية المتطفلة على اسماك الكارب في } \\
& \text { مزرعة اسماك الفرات، محافظة بابل، العراق. أطروحة دكتور اه، كلية العلوم، جامعة بابل: إكـ ال صفحة. }
\end{aligned}
$$

